\title{
Half metals: from formal theory to real material issues
}

\author{
Warren E Pickett ${ }^{1}$ and Helmut Eschrig ${ }^{2}$ \\ ${ }^{1}$ Department of Physics, University of California Davis, Davis, CA 95616, USA \\ 2 IFW Dresden, PF 270 116, D-01171 Dresden, Germany
}

Received 21 November 2006, in final form 23 January 2007

Published 3 July 2007

Online at stacks.iop.org/JPhysCM/19/315203

\begin{abstract}
At the basic level of collinear spin density functional theory, half metallic ferromagnets represent a fundamentally different state of matter: for lowenergy physics the spin degree of freedom is absent, although the system is spin polarized. This makes such systems highly attractive for spintronics applications, but also introduces fundamental new phenomena such as a superconducting state in which the concept of 'spin-pairing' never appears. A fully relativistic theory introduces spin-orbit coupling and destroys the precise aspect of half metallicity; does this make 'half metals' a half truth? Obviously not in any real sense: spin-orbit coupling arises as a perturbative effect, and although necessitating reconsideration from the formal viewpoint, leaves half metallicity as a qualitatively distinct state. We provide a simple model that suggests that in appropriate circumstances this qualitative distinction may even survive strong spin-orbit coupling.
\end{abstract}

\section{Background}

Charge and spin transport in solids is due to valence electrons. If one abstracts from the other dynamical degrees of freedom (motion and internal excitations of the lattice ions, the influence of which in transport problems can be subsumed into relaxation times for electron motion) the model to be considered is an interacting fermion system in a crystalline background. There are several universality classes of the ground state and the low-lying excitation spectrum of this model. They are distinguished by (i) a two-dimensional Fermi surface (FS) in threedimensional momentum space, (ii) Fermi lines instead, (iii) Fermi points, and (iv) a gapped ground state separated from the excitations by an energy gap $\Delta$. In the first three cases there are gapless excitations with linear dispersion in the vicinity of the FS (figure 1). Class (i) consists of metals, classes (ii) and (iii) comprise semi-metals and unconventional superconductors, and class (iv) contains the semiconductors and the conventional superconductors. Of course, superconductors are also distinct in another respect, they are Weyl-Bogoliubov rotated in the charge-particle space from pure particle and hole excitations into mixed ones (the BogoliubovValatin transformation). While the universality classes (i), (iii) and (iv) are robust with respect to changes in the interaction strength, (ii) is in general not [1]. 


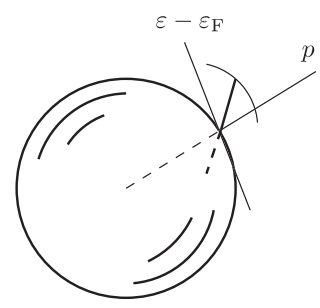

(a)

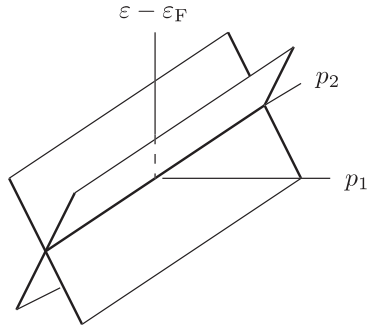

(b)

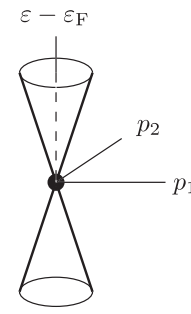

(c)

Figure 1. Dispersion relations of (semi-)metals of (a) class (i), (b) class (ii) and (c) class (iii). See also the text.

For light elements up to nuclear charge $Z \approx 30$ in particular, but often more generally, spin-orbit coupling of the valence electrons can be treated as a weak perturbation and can be neglected in qualitative and even semi-quantitative treatment. Then one is left with two spin subsystems which are only coupled by a common chemical potential. (In practice, this coupling comes from some residual spin-orbit interaction or from spin-flip scattering on the other degrees of freedom.) It is only this latter model which allows one to speak of half metallicity: one spin subsystem is a metal and the other is an insulator or semiconductor (i.e. gapped) or a semi-metal (zero-gapped). This well characterized model system has a number of characteristic properties, interesting as such and/or relevant to applications: namely, absence of magnetic Pauli susceptibility and full spin polarization of conduction electrons, to mention the two most basic ones. Adding more interactions and more degrees of freedom to the model will change these characteristics into approximate properties of real materials, and these changes can be estimated perturbatively.

\section{Brief phenomenology of half metals}

\subsection{Most relevant material parameters}

The most direct parameters of an ideal half metal are the excitation gaps in the various spin channels, shown schematically in figure 2. In this figure, the density of states (DOS) of the electron excitation spectrum is shown with the hole states shadowed. The spin-flip excitation gaps are $\Delta_{\downarrow \uparrow}$ for a transition from minority to majority spin (increase of magnetization), $\Delta_{\uparrow \downarrow}$ for a transition from majority to minority spin (decrease of magnetization) and the gap $\Delta_{\downarrow \downarrow}=\Delta_{\downarrow \uparrow}+\Delta_{\uparrow \downarrow}$ for spin-diagonal excitations of minority-spin states. The majorityspin-diagonal channel is metallic and hence gapless. Because of the spin-flip gaps, the Pauli susceptibility would clearly be zero in this situation. These characteristics apply exactly only at zero temperature; temperature fluctuations degrade true half metallicity [2,3].

Accidentally, one of the spin-flip gaps, $\Delta_{\downarrow \uparrow}$ or $\Delta_{\uparrow \downarrow}$, may be zero, and the Pauli susceptibility would be anisotropic, being zero for fields in one polarization direction and nonzero (but possibly of higher order due to the zero minority-spin DOS) in the opposite field direction. At least theoretically there also exists a possibility that all three of these gaps are zero, while $\mathrm{DOS}_{\downarrow}$ is still equal to zero and there is a Fermi point with conical dispersion (figure 1(c)) in the minority-spin band. In this case the higher than first order Pauli susceptibility is nonzero for both directions of the field. Finally, complementary cases may exist where the role of majority and minority spin is interchanged. 


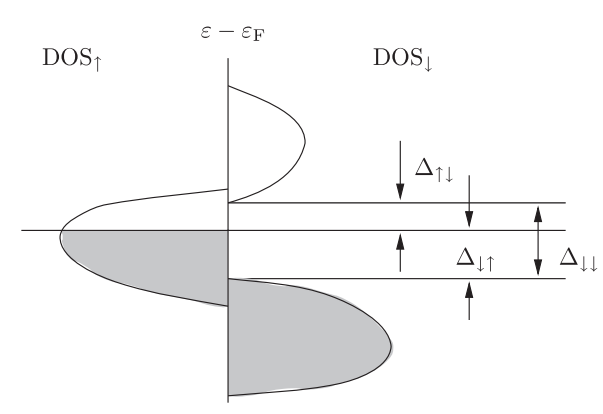

Figure 2. Schematic spin-resolved DOS of the excitation spectra of a half metal with the spin-flip gaps $\Delta_{\uparrow \downarrow}$ and $\Delta_{\downarrow \uparrow}$, and spin-conserving gap $\Delta_{\downarrow \downarrow}$ equal to their sum.

From a technological point of view, one strong interest in half metals arises from their potential as a spin injector in spintronics [4-6]. To quantify their usefulness, a generalized definition of the degree of spin polarization [7]

$$
P_{X}=\frac{\left\langle P_{\uparrow} X P_{\uparrow}\right\rangle-\left\langle P_{\downarrow} X P_{\downarrow}\right\rangle}{\left\langle P_{\uparrow} X P_{\uparrow}\right\rangle+\left\langle P_{\downarrow} X P_{\downarrow}\right\rangle}
$$

of the observable $X$ becomes relevant, where $P_{\uparrow}$ is the projector on the up-spin sector of the state space. The simplest case is $X=$ DOS, which in the above simplified (but conventional) considerations for a half metal leads to $P=1$ (or 100\%). In reality, due to neglected further interactions, notably spin-orbit coupling ([8], and see below), this is never precisely the case. Moreover, depending on whether the electron transport is metallic or a tunnelling process, the quasi-particle DOS or the single-particle DOS, respectively, is relevant. Tunnelling processes are due to wavefunction overlap, and hence the corresponding DOS contains the square of the wavefunction renormalization constant $(Z)$. In metallic transport, on the other hand, one quasi-particle carries an unrenormalized full quantum of charge due to the backflow in the polarization cloud. Hence, the wavefunction renormalization constant does not enter as is the case for the quasi-particle DOS. However, it is not simply the DOS itself which matters in metallic transport because the velocity in the direction of transport is important. Depending on whether it is diffusive or ballistic at the interface, $X=\mathrm{DOS} \cdot \boldsymbol{v}^{2}$ or $X=\mathrm{DOS} \cdot \boldsymbol{v}$, respectively, is the quantity that enters the conductivity, where $v$ is the quasi-particle velocity on the FS.

\subsection{Classes of half metals}

The concept of a half metallic electronic structure is a straightforward one, but within this broad specification of conduction in only one of the two spin channels there can be very interesting and potentially important distinctions. On the simplest level one can readily realize that the conducting channel can be either the majority-spin, or the minority-spin, channel. The other simple distinction is that, among the various integer values of the spin moment $M$ per cell that may occur, the special case is $M=0$, dubbed the half metallic antiferromagnet. This type of material is discussed in more detail below.

Other aspects that arise are (1) the characteristics of conduction: itinerant, versus the more complex (moderately or strongly) correlated case, and (2) the characteristics of the nonconducting channel. There may be a simple band-like gap in the non-conducting channel, a correlation-induced gap or a zero-gap (semi-metal). The variety of possibilities that can be anticipated, and in several cases identified with some degree of confidence, have been tabulated and discussed by Coey and collaborators $[9,10]$. Transitions between these various cases can 
be effected in the laboratory, for example the transformation from half metal to semiconductor via pressure [11] as the counterpart of a metal-to-semiconductor (or insulator) transition.

\section{Spin density functional theory of half metallicity}

Conventional non-relativistic spin density functional theory (SDFT) [16] is a many-body approach to a model of interacting electrons with spin-independent interaction and a spindiagonal kinetic energy operator, in an external spin-independent potential and possibly an external magnetic field which couples to the electron spin only and not to the orbital motion. For systems containing light elements up to the $3 \mathrm{~d}$ metals this is often a reasonable model. In the absence of a magnetic field or in a homogeneous magnetic field the direction of which is chosen as the spin-quantization direction, its ground state is an eigenstate of the total $z$ component of the spin $S_{z}=\sum_{i=1}^{N} s_{z}^{i}$, or in case of degeneracy may be chosen to be such a state. Hence it is spin-collinear, and half metallicity may be a rigorous issue. This is the situation that was analysed in [12].

\subsection{Formal issues}

It is worth reviewing very briefly the formal state of the theory for the spin degree of freedom in DFT.

- Hohenberg and Kohn [13] provided a proof for scalar potentials that there is a unique mapping $n \rightarrow(v \bmod$ constant $)$ from ground state particle densities $n$ to potential classes coinciding up to a constant.

- In their proof they assumed without specific mention (by saying that wavefunctions for different potentials are obviously different) that only those potentials are considered whose wavefunctions do not vanish on a domain of non-zero measure. Lieb [14] assigned a functional space of such potentials.

- For the case of spin-dependent potentials, the simplest result to visualize is the following: for collinear spin densities, there is a mapping $\left\{n_{s}\right\} \rightarrow\left(\left\{v_{s} \bmod\right.\right.$ constant $\left.\left.C_{s}\right\}\right)$ [12], that is, a non-zero constant magnetic field $B_{z}=\left(C_{\uparrow}-C_{\downarrow}\right) / \mu_{\mathrm{B}}$ may not affect the spin densities. This specifies the case of a half metallic ferromagnet, and specific examples have been discussed [15].

- For non-collinear spin densities $n_{\alpha \beta}$, the existing analysis [12] allows the possibility that the spin density is unchanged by certain inhomogeneous external fields. A case satisfying the necessary criteria is hard to imagine on the microscopic (atomic) scale.

\subsection{Consequences of half metallicity}

Within this frame one solves separately Kohn-Sham equations for spin-up and spin-down subsystems. Typically this is done by varying the spin imbalance to minimize the energy (assuming some spin-equilibrium interaction, as noted above), which then gives the ground state charge density, spin density and energy. One may also restrict the space to given electron numbers $N_{\uparrow}$ and $N_{\downarrow}$. This 'constrained spin density functional' approach is commonly referred to as a fixed spin moment calculation, with total spin magnetic moment given by (using the spin $g$-factor $g_{s}=2$ neglecting tiny quantum electrodynamic radiation corrections)

$$
M=\mu_{\mathrm{Bohr}}\left(N_{\uparrow}-N_{\downarrow}\right)
$$

with the Bohr magneton $\mu_{\text {Bohr }}$. The resulting difference in the chemical potentials, divided by the Bohr magneton, is the applied homogeneous magnetic field corresponding to the solution; 
that is, one obtains $B=B(M)$. In a normal metal one varies $M$ by varying $N_{\uparrow}$ and $N_{\downarrow}$ to find the spontaneous moment $M(B=0)$. This is given by the numbers $N_{\uparrow}$ and $N_{\downarrow}$ for which the chemical potentials in the two Kohn-Sham equations are equal.

We consider the precise case of a stoichiometric compound, thus the number of valence electrons is an integer. (Alloys with a non-integral mean number of valence electrons can be handled approximately but rather accurately.) If one of the spin subsystems, the majority-spin system say, has an energy gap at the chemical potential, then the corresponding number $N_{\uparrow}$ must be an integer corresponding to the number of filled bands; this leaves an integer also for the opposite spin, and hence also for the difference, which is $M / \mu_{\mathrm{Bohr}}$. The position of the chemical potential at zero temperature within the gap has no effect on the ground state (wavefunction, or density); an external homogeneous magnetic field is arbitrary within an interval corresponding to the gap [12]. A change of $B$ within that interval therefore does not change $M$ : as a result, a half metal with a non-zero gap has zero Pauli susceptibility, and this can be used (in principal, at least) for experimental verification of half metallicity. This is not true for the special case of an ideal half metal with a Fermi point in one spin channel. In that case $B(M)$ is unique, but the linear Pauli susceptibility still vanishes. Instead, $M(B)$ has an inflection point with a horizontal tangent.

The SDFT treatment of half metallicity suffers from the same gap problem as the DFT for semiconductors. The difference between the external potential and the chemical potential (chemical potentials for the spin subsystems), which is the functional derivative of the density functional (spin density functional [16]) is not uniquely defined in the direction of the functional space corresponding to a change of $N\left(N_{\uparrow}, N_{\downarrow}\right)$. Euler's equations can only be applied to the variational problem under the constraint that these numbers are fixed. Thus this condition is not resolved by the approach of [17]. One can resort, fortunately, to fixed moment calculations in the case of half metals, where the derivative of the spin density functional (the spin-dependent XC potential) makes a jump across the integer number of $M / \mu_{\text {Bohr }}$ [12]. Local-density approximations cannot make such a jump and hence fail in principle to reproduce those gaps correctly, and also the corresponding intervals of chemical potential or $B$-field. Nevertheless, local spin density functional (LSDA) in most cases correctly obtains the half metallic state (with a reduced gap) as in most cases it also correctly obtains the semiconducting state (with a reduced gap). LSDA $+U$ is sometimes tried instead; but this should be considered with reservations in case of itinerant systems.

\section{Half metallic antiferromagnets}

The peculiarly named half metallic antiferromagnets are the subset of stoichiometric half metals in which the integer moment per cell corresponds to the integer zero. In truth, they are half metallic ferrimagnets in which the sum of the down spins exactly cancels the sum of the up spins, and are better characterized as compensated half metals (CHM), a simplification of a designation introduced by the Mainz group [18]. Certainly there is no antiferromagnetic symmetry. Still, the concept of a material with dense magnetic moments and the symmetry of a ferromagnet, yet with no macroscopic field, is an intriguing one with potentially profound implications. Most obviously, the current would be $100 \%$ spin-polarized without the materials displaying any macroscopic magnetic field. Such a property would provide the functionality of a HM ferromagnet but enable different characteristics in devices (being impervious to magnetic fields in the environment, for example).

The CHM provides the platform for a novel state of matter, called the single-spin superconductor (SSS) [19]. There are now a few examples of superconductivity coexisting with robust ferromagnetism, with $\mathrm{UGe}_{2}$ (under pressure) being the clearest example. The 
character of the allowed pairing states in such systems has been characterized, the interesting question being how the spin state of the Cooper pair (necessarily spin-parallel pairing) couples with the orbital state to produce order parameters with exotic flavours [20]. On the GinzburgLandau level of description of currents, it is necessary that the superconducting phase appears in concert with an accompanying flux lattice, to accommodate the intrinsic magnetic field present in the ferromagnet. The SSS system is distinct: the spin degree of freedom is simply missing from consideration (much below the magnetic ordering temperature, at least). The result is the superconducting pairing of spinless fermions, with allowed order-parameter symmetries having been worked out for common crystal structures [21].

Thus from both the pure science aspect and the standpoint of applications, this CHM possibility presents a novel system, deserving designation as a 'new state of matter'. The CHM concept was introduced by van Leuken et al, who provided a structurally and electronically complex example [22]. It was pointed out not long after that the phenomenon of anti-alignment of moments with identical values of spin (spin $3 / 2$, say, corresponding to $3 \mu_{\mathrm{B}}$ on an ion) should not be so unlikely in common crystal structures [24]. The double perovskite structure (elpasolite, to solid state chemists) $\mathrm{A}_{2} \mathrm{XZO}_{6}(\mathrm{~A}=$ cation; $\mathrm{X}, \mathrm{Z}$ are magnetic ions) is a good place to start, since there are a great many members of this class already reported by Anderson et al [23], including several with two transition metal ions (which are likely to provide magnetic moments). The perovskite structure should also be favoured for CHMs because of the tendency for antiferromagnetic superexchange in the structure.

An early suggestion for a favourable compound [24], calculated to have compensating $S=1$ spins on the transition metal ions, was $\mathrm{La}_{2} \mathrm{MnVO}_{6}$. Local spin density calculations led to anti-aligned $\mathrm{Mn}^{3+}\left(\mathrm{d}^{4}\right)$ and $\mathrm{V}^{3+}\left(\mathrm{d}^{2}\right)$ ions, with $\mathrm{Mn}$ in the low-spin state. Efforts to synthesize this compound have not produced a well ordered material [25], so the suggestion has not really been definitively tested; achieving well ordered compounds is one of the challenges in the double perovskite class of materials. The indications are indeed that both $\mathrm{Mn}$ and $\mathrm{V}$ are trivalent, and that exchange coupling is strongly antiferromagnetic in sign. The Curie-Weiss moment suggests that $\mathrm{Mn}$ assumes the high-spin state $S=2$ rather than $S=1$, destroying the possibility of being a CHM and instead resulting in a ferrimagnet.

Subsequently the possibility of a CHM state in the double perovskite system $\mathrm{LaAVRuO}_{6}$ was proposed [26]. Perhaps motivated by the observation that LSDA predicted a low-spin $\mathrm{Mn}^{3+}$ ion in $\mathrm{La}_{2} \mathrm{MnVO}_{6}$ whereas magnetic data point to a high-spin ion, they applied the correlated band theory LDA $+U$ method. In such a $3 d-4 d$ pair system, site ordering of the ions may be promoted due to size difference. They concluded that this system provides strong candidates for CHM materials. We are not aware of experimental attempts to test these predictions. The theoretical search for CHMs was extended to the system $\mathrm{LaAVMoO}_{6}, \mathrm{~A}=\mathrm{Ca}, \mathrm{Sr}, \mathrm{Ba}$ [27]. Powder samples of some members of this system had been synthesized and characterized earlier [28]. Although the desired AF coupling was obtained and several examples of HM character (or nearly so) resulted, they obtained ferrimagnetic states rather than CHM states.

The latest promising possibility is in the Heusler system, with the compound $\mathrm{Mn}_{3} \mathrm{Ga}$ calculated by the Mainz group [18] to be very close to a CHM. The Mn moment of $3.03 \mu_{\mathrm{B}}$ on the $\mathrm{Y}$ site of the $\mathrm{X}_{2} \mathrm{YZ}$ structure is compensated by the two oppositely directed moments of $-1.54 \mu_{\mathrm{B}}$ (and a small induced Ga moment). The uppermost part of the minority valence band at $\Gamma$ (the band at the lower side of the gap) crosses the Fermi level by $\sim 0.1 \mathrm{eV}$ in LDA calculations, so the polarization at $E_{\mathrm{F}}$ is not quite $100 \%$. Because the bandgap is underestimated in LDA (the amount is not known), this system holds the possibility of being a CHM. The ordered alloy $\mathrm{Mn}_{2}\left(\mathrm{Mn}_{0.5} \mathrm{~V}_{0.5}\right) \mathrm{Si}$ is also calculated to be a CHM [18].

In real materials, with their additional degrees of freedom, other intriguing scenarios can be envisioned. When f-elements are involved, large orbital moments may coexist with spin 


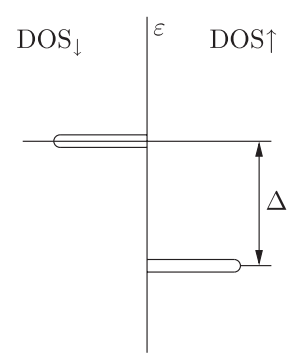

(a)

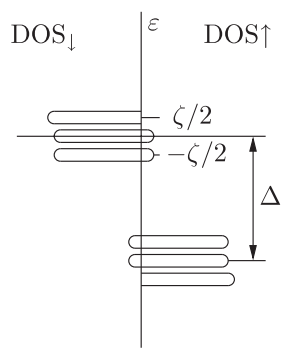

(b)

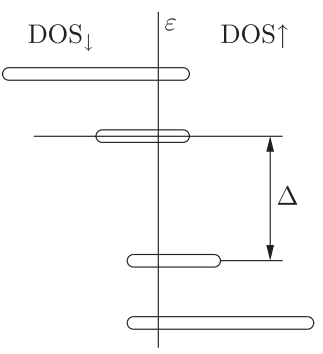

(c)

Figure 3. Spin-resolved density of states (DOS) of a spin split $\mathrm{t}_{2 \mathrm{~g}}$ complex: (a) without $l \cdot s$ coupling and (b) weak $l \cdot s$ coupling, splittings are of order $\zeta / \Delta$. (c) Spectrum with strong $l \cdot s$ coupling $\zeta \equiv \Delta$ (see text for further explanation).

moments and may cancel the spin moment to produce an 'antiferromagnet' result for the total moment, which again could be half metallic (conducting for one direction of the spin only) and could have the advantages of a CHM.

\section{Effect of spin-orbit coupling}

Spin-orbit coupling $(l \cdot s$ coupling) mixes the $\uparrow$ and $\downarrow$ components of the Bloch states and hence impairs half metallicity. If the spin-orbit coupling strength $\zeta$ is weak, the spin hybridization is expected to be proportional to $\zeta$, and the degree of spin polarization is reduced according to the DOS ratio at the Fermi level $\operatorname{DOS}_{\uparrow} / \mathrm{DOS}_{\downarrow} \sim \zeta^{2}$. In order to quantify the effect in more detail, we consider the model pictured in figure $3(\mathrm{a})$.

Assume a narrow degenerate $t_{2 g}$ complex of d-states close to the Fermi level and spin polarized such that the majority $(\uparrow)$ band is filled and the minority $(\downarrow)$ band holds less than one electron per magnetic atom, i.e. $\mathrm{a} \mathrm{d}^{3+\epsilon}$ ion with $0<\epsilon<1$. The $\mathrm{e}_{\mathrm{g}}$ bands are assumed to be so far away in energy (for instance by covalency splitting with six oxygen neighbours) that they can be removed from consideration. The spin-orbit Hamiltonian is

$$
H_{l \cdot s}=\frac{\zeta}{2}\left(2 l_{z} s_{z}+l_{+} s_{-}+l_{-} s_{+}\right)
$$

The $\mathrm{t}_{2 \mathrm{~g}}$ complex is built of real tesseral harmonics

$$
\begin{aligned}
& |x y\rangle=\frac{|+2\rangle-|-2\rangle}{\mathrm{i} \sqrt{2}}, \\
& |x z\rangle=\frac{|+1\rangle+|-1\rangle}{\sqrt{2}}, \\
& |y z\rangle=\frac{|+1\rangle-|-1\rangle}{\mathrm{i} \sqrt{2}},
\end{aligned}
$$

where $|m\rangle$ are $l_{z}$ eigenstates with quantum number $m$ of $l=2$. It is easily found that the $6 \times 6$ Hamiltonian matrix of the spin-dependent $\mathrm{t}_{2 \mathrm{~g}}$ states splits into two $3 \times 3$ blocks, one of which is

$$
H_{l \cdot s}=\left(\begin{array}{lll}
|x y \uparrow\rangle & |x z \downarrow\rangle & |y z \downarrow\rangle
\end{array}\right)\left(\begin{array}{ccc}
-\Delta & -\mathrm{i} \frac{\zeta}{2} & \frac{\zeta}{2} \\
\mathrm{i} \frac{\zeta}{2} & 0 & -\mathrm{i} \frac{\zeta}{2} \\
\frac{\zeta}{2} & \mathrm{i} \frac{\zeta}{2} & 0
\end{array}\right)\left(\begin{array}{c}
\langle x y \uparrow| \\
\langle x z \downarrow| \\
\langle y z \downarrow|
\end{array}\right) .
$$


This subspace involves one of the three majority-spin states and two of the three minority-spin states. The remaining states form the other $3 \times 3$ block which is obtained simply by a sign change of the matrix elements and then a down shift of the diagonal by $\Delta$.

It is instructive to note that in the angular momentum basis of $l_{z}= \pm 1$ minority states the Hamiltonian matrix becomes

$$
\tilde{H}_{l \cdot s}=\left(\begin{array}{lll}
|x y \uparrow\rangle & |+1 \downarrow\rangle & |-1 \downarrow\rangle
\end{array}\right)\left(\begin{array}{ccc}
-\Delta & 0 & \frac{\zeta}{\sqrt{2}} \\
0 & \frac{\zeta}{2} & 0 \\
\frac{\zeta}{\sqrt{2}} & 0 & -\frac{\zeta}{2}
\end{array}\right)\left(\begin{array}{c}
\langle x y \uparrow| \\
\langle+1 \downarrow| \\
\langle-1 \downarrow|
\end{array}\right) .
$$

In this subspace the $l_{z}=+1$ state decouples from the spin-orbit problem. Since the common interpretation is in terms of real $\mathrm{t}_{2 \mathrm{~g}}$ states, we return to that basis to follow the consequences.

Consider first the case $\zeta \ll \Delta$ of weak spin-orbit coupling compared to the exchange splitting. To leading order in $\zeta / \Delta$, the eigenvalues are easily obtained to be $\varepsilon= \pm \zeta / 2$ and $\varepsilon=-\Delta$. The corresponding eigenstates have the form

$$
\phi=|x y \uparrow\rangle a+|x z \downarrow\rangle b_{x}+|y z \downarrow\rangle b_{y}
$$

with the eigenstate amplitudes $\left\{a, b_{x}, b_{y}\right\}$ given, to within a normalization constant, by

$$
\begin{aligned}
& \phi_{+\zeta / 2}:\{0,1, \mathrm{i}\} \\
& \phi_{-\zeta / 2}:\left\{\frac{\zeta}{\Delta}, \mathrm{i}, 1\right\}, \\
& \phi_{-\Delta}:\left\{1, \frac{\zeta}{2 \Delta} \mathrm{i}, \frac{\zeta}{2 \Delta}\right\} .
\end{aligned}
$$

These states have $\left\langle l_{z}\right\rangle=+1,-1+(\zeta / 2 \Delta)^{2},-(\zeta / 2 \Delta)^{2}$, respectively.

The two minority-spin states split (equation (6)), and the lower one (like the majority-spin state) gets spin-mixed by a fraction of order $\zeta / \Delta$. In the same way, the other block yields a splitting of the two majority-spin states and a spin hybridization of the two upper states in energy. The result is summarized in figure 3(b). For our assumed filling, the Fermi level is pinned in the lowest minority-spin state, and the DOS ratio is

$$
\frac{\mathrm{DOS}_{\uparrow}}{\mathrm{DOS}_{\downarrow}}=\frac{\zeta^{2}}{2 \Delta^{2}}
$$

which is the anticipated result for this case. However, the result that in the case of less than one hole in the minority bands, and hence a pinning of the Fermi level in the uppermost state, the impairing of half metallicity is of higher than second order in the ratio $\zeta / \Delta$ is not anticipated.

Next, the particular case $\zeta=\Delta$ is considered as an example of strong spin-orbit coupling. Now, the eigenvalues of (5) are $\varepsilon=\Delta / 2, \varepsilon=0$ and $\varepsilon=-3 \Delta / 2$. The corresponding states (up to a normalization constant) are

$$
\begin{aligned}
& \phi_{\Delta / 2}:\{0,1, i\} \\
& \phi_{0}:\{1, i, 1\} \\
& \phi_{-3 \Delta / 2}:\left\{1,-\frac{i}{2},-\frac{1}{2}\right\}
\end{aligned}
$$

with $\left\langle l_{z}\right\rangle=+1,-1 / 2$ and $-1 / 2$, respectively. Again, the uppermost state has pure spin $\downarrow$ and orbital +1 character, while now the two lower ones are strongly spin-entwined. However, now the other $3 \times 3$ Hamiltonian block yields strongly hybridized upper states and the lowest non-hybridized, where the highest and lowest in energy of all six states is doubly degenerate (figure 3(c)). For the DOS ratio at Fermi level one has

$$
\frac{\operatorname{DOS}_{\uparrow}}{\operatorname{DOS}_{\downarrow}}=\frac{1}{2}
$$


in our case of less than one electron in the minority states, and half this value (due to the degeneracy with a non-hybridized state) in the case of less than one hole. In any case half metallicity is more or less completely destroyed.

Unexpectedly, even in this case of strong spin-orbit coupling, there is for certain geometries a band which does not spin hybridize. Moreover (or rather, as a consequence) there is an associated large contribution to the orbital moment. Considering the above degeneracy of states as a particular model feature, one consequence is that strong spin-orbit coupling even with large orbital moments does not necessarily destroy half metallicity, so materials with heavy elements as components are not completely ruled out as candidates for half metallicity, as simple heuristic arguments ('common sense') would suggest. For every structure and band filling a relativistic band calculation is needed to decide this question.

The influence of $l \cdot s$ coupling in the case of a half metal with a Fermi point and a conically dispersed band in its neighbourhood is similar. (Slightly non-stoichiometric $\mathrm{Ag}_{2} \mathrm{Te}$ could be a semi-metallic non-polarized case with a Fermi point [29].) This situation needs the degeneracy of a band crossing at the Fermi level. It is a general result that $l \cdot s$ coupling removes symmetry derived band degeneracies. If the (non-relativistic) conical dispersion around a Fermi point is symmetry related, $l \cdot s$ coupling will open a gap and thus remove the Fermi point completely. However, again bands of different symmetry group representations may cross at arbitrary points in momentum space, and if such a crossing of a majority- and a minority-spin band happens at the Fermi level it probably survives $l \cdot s$ coupling.

Orbital magnetic polarization (orbital moments) is another issue closely related to $l \cdot s$ coupling. Magnetic polarization in a solid is normally triggered by the spin dependence of exchange interaction. Its presence then induces an orbital polarization which otherwise would be quenched by covalency, and is further enhanced by exchange and correlation effects (second Hund's rule coupling) [30]. The underlying mechanism is the shifting of spectral weight across the Fermi level, and in the case of half metallicity shifting across the half metal gap without necessarily changing much the degree of spin polarization at the Fermi level. A related consequence is that in the presence of $l \cdot s$ coupling the occupation of the spin states is no longer an integer, so the Pauli susceptibility no longer vanishes. A thorough SDFT analysis along the above lines of Heusler alloys has been provided recently [31].

\section{Summary}

It has become accepted that the half metallic ferromagnetic state is a fundamentally different state from that of a normal ferromagnet. Here we have looked briefly at this question from a few different viewpoints. First, the formal underpinnings of the spin density functional theory of half metals were outlined. Then, the relativistic effect of spin-orbit coupling was considered (in principle it destroys half metallicity, in practice it is a minor effect for the smaller magnetic atoms $(Z \sim 30$ or less)). A model treatment was given to show that it is possible even for heavier atoms, with an appropriate structure and band filling, that a half metal (or near) state may survive, and may even have significant orbital moment.

Thirdly, we revisited the possibility of a half metal with zero net moment, the so-called half metallic antiferromagnet, with the better terminology being a compensated half metal. Although there are several known or suspected half metals with various values of (integer) moment, there is as yet no good prospect of a compensated half metal with $M=0$. The compound $\mathrm{Mn}_{2}$ Ga predicted by the Mainz group [18], while not yet synthesized, is an excellent example of the possibilities that exist even in very common crystal structures. Given the substantial number of ferrimagnets in intermetallic compounds and oxides (such as the double perovskites), there is good reason to anticipate that half metallic antiferromagnets lurk within 
known systems waiting to be discovered. A new kind of superconductivity is possible in such systems, somewhat analogous to superconductivity in a ferromagnet but with the minority bands having 'evaporated' taking their own superconducting gap with them.

\section{Acknowledgments}

We acknowledge recent important interactions on the theory and properties of half metals with K Koepernik, M Richter and M Sargolzaei. One of the authors (WEP) was supported partially by the US Department of Energy's Stewardship Science Academic Alliances Program, and both authors have benefited from the support of the Alexander von Humboldt Foundation.

\section{References}

[1] Volovik G E 2003 The Universe in a Helium Droplet (Oxford: Clarendon) see also Horav̂a P 2005 Phys. Rev. Lett. 95016405

[2] Skomski R and Dowben P A 2002 Europhys. Lett. 58544

[3] Dowben P A and Skomski R 2003 J. Appl. Phys. 937948

[4] Wolf S A, Awschalom D D, Buhrman R A, Daughton J M, von Molnar S, Roukes M L, Chtchelkanova A Y and Treger D M 2001 Science 2941488

[5] Žutić I, Fabian J and Sarma S D 2004 Rev. Mod. Phys. 76323 and references therein

[6] Pickett W E and Moodera J S 2001 Phys. Today 5431

[7] Mazin I I 1999 Phys. Rev. Lett. 831427

[8] Mavropoulos P, Sato K, Zeller R, Dederichs P H, Popescu V and Ebert H 2004 Phys. Rev. B 69054424

[9] Coey J M D and Chien C L 2003 MRS Bull. 28720

[10] Coey J M D and Sanvito S 2004 J. Phys. D: Appl. Phys. 37988

[11] Duan C-G, Sabiryanov R F, Liu J, Mei W N, Dowben P A and Hardy J R 2005 Phys. Rev. Lett. 94237201

[12] Eschrig H and Pickett W E 2001 Solid State Commun. 118123

[13] Hohenberg P and Kohn W 1964 Phys. Rev. 136 B864

[14] Lieb E H 1983 Int. J. Quantum Chem. 24243

[15] Capelle K and Vignale G 2001 Phys. Rev. Lett. 865546

[16] von Barth U and Hedin L 1972 J. Phys. C: Solid State Phys. 51629

[17] Argaman N and Makov G 2002 Phys. Rev. B 66052413

[18] Wurmehl S, Kandpal H C, Fecher G H and Felser C 2006 J. Phys.: Condens. Matter 186171

[19] Pickett W E 1996 Phys. Rev. Lett. 773185

[20] Sigrist M and Ueda K 1991 Rev. Mod. Phys. 63239

[21] Rudd R E and Pickett W E 1998 Phys. Rev. B 57557

[22] van Leuken H and de Groot R A 1995 Phys. Rev. Lett. 741171

[23] Anderson M T, Greenwood K B, Taylor G A and Poeppelmeier K R 1993 Prog. Solid State Chem. 22197

[24] Pickett W E 1998 Phys. Rev. B 5710613

[25] Androulakis J, Katsarakis N and Giapintzakis J 2002 Solid State Commun. 12477

[26] Park J H, Kwon S K and Min B I 2002 Phys. Rev. B 65174401

[27] Park M S and Min B I 2005 Phys. Rev. B 71052405

[28] Uehara M, Yamada M and Kimishima Y 2002 Solid State Commun. 129385

[29] Abrikosov A A 1998 Phys. Rev. B 582788

[30] Eschrig H, Sargolzaei M, Koepernik K and Richter M 2005 Europhys. Lett. 72611

[31] Sargolzaei M, Richter M, Koepernik K, Opahle I, Eschrig H and Chaplygin I 2006 Phys. Rev. B 74224410 